\title{
Biomimetic hydrophilic foam with micro/nano-scale porous hydrophobic surface for highly efficient solar-driven vapor generation
}

\author{
Yeran $\mathrm{Li}^{1 \dagger}$, Xin Jin ${ }^{2 \dagger}$, Wei $\mathrm{Li}^{1}$, Jiarong $\mathrm{Niu}^{1}$, Xing $\mathrm{Han}^{1}$, Xiaofang Yang ${ }^{1}$, Wenyu Wang ${ }^{1^{*}}$, Tong Lin ${ }^{1,3}$ and \\ Zhengtao $\mathrm{Zhu}^{1,4^{*}}$
}

\begin{abstract}
Solar-driven vapor generation by localized solar heating of a photothermal material is an environmentally friendly approach for seawater desalination and wastewater purification. In this work, inspired by the leaf transpiration in nature, we designed a novel bionic leaf to realize highly efficient solar vapor generation. The leaf-inspired three-dimensional (3D) material structure had a hydrophilic polyvinyl alcohol (PVA) foam layer (equivalence to the mesophyll tissue layer in a leaf) with high porosity and low thermal conductivity, a photothermal polypyrrole (PPy) layer (equivalence to the chlorophyll layer in a leaf) coated on the PVA foam, and a micro/nano-scale porous hydrophobic surface layer (equivalence to the stomal layer in a leaf). The PVA network had microchannels for water transportation and reducing water evaporation enthalpy, the PPy layer absorbed and converted solar energy to heat water locally, and the hydrophobic porous surface layer enhanced the rate of vapor escape. The asfabricated vapor generator based on the bionic-leaf structure achieved an impressive high evaporation rate of $3.09 \mathrm{~kg} \mathrm{~m}^{-2} \mathrm{~h}^{-1}$ under one sun irradiation $\left(1 \mathrm{~kW} \mathrm{~m}^{-2}\right)$. The prototype vapor generator desalinated and purified brine and seawater successfully under natural sunlight. Such solar vapor generator based on the biomimetic structure provides a potential low-cost and highly efficient water purification technology to help mitigate the global water crisis using sustainable energy.
\end{abstract}

Keywords: bionic leaf structure, solar thermal, interfacial evaporation, solar desalination, water purification

\section{INTRODUCTION}

Demands for clean water in industry, agriculture and household have surged due to the increase of population and improvement of living standards [1]. Solar vapor generation as a green and sustainable technology without using extra energy has great potential in water purification and treatment due to the abundant solar energy supply on earth [2]. While the conventional solar stills have an efficiency of $30 \%-50 \%$, the recently devel- oped interfacial solar vapor generation using photothermal materials can achieve more than $90 \%$ solar vapor conversion efficiency [3-7]. It is worth noting that in order to improve the performance of solar desalination, methods using extra energy resources (e.g., photovoltaic, ground heat) have been reported to facilitate high-performance solar desalination [8-10].

In the interfacial solar vapor generation, the photothermal material coated on the porous structure floating on the water surface converts the absorbed solar energy into heat and evaporates water in the porous channels. In order to improve the efficiency of solar vapor generation, four factors have to be optimized, including the efficient absorption of solar energy, energy/thermal management, continuous water transportation, and prompt water vapor diffusion through the structured channels. Considering these four factors, many researchers have explored advanced materials and innovative structures, including inspiration from the natural world, to design efficient solar evaporators [11-16]. For example, noble-metal nanoparticles [12] and carbon-based materials (e.g., carbon black [13], graphene [14], and carbon nanotubes [15]) have high solar absorptance, which can increase the solar-thermal conversion efficiency of a solar evaporator to 70\%-90\%. Photothermal polymers with tunable molecular structures can be synthesized to have superior solar absorptance (up to 99\%) as well as good adhesion to the interfacial porous material for solar vapor generation [16]. For energy/thermal management and continuous water supply, researchers have demonstrated various structures ranging from two-dimensional (2D) structures (e.g., film [17], paper [18], felt [19], and fabric [20]) to 3D structures/ configuration [21-25] (e.g., aerogel [26], hydrogel [27], foam [28], and sponge [29]). Furthermore, structures derived from natural resources including woods [30], corns [31], and mushrooms [32] have been developed for efficient solar evaporation systems. There are several reports on the surface wettability treatment of the solar evaporation material to balance water transport/supplementary/distribution and evaporation [33-35], and to enhance the durability [36,37]. For example, hydrophobic patches were formed on the surface of hydrophilic hydrogel to increase the water layer thickness in the hydrophilic regions and

\footnotetext{
${ }^{1}$ State key laboratory of Separation Membranes and Membrane Processes, School of Textile Science and Engineering, Tiangong University, Tianjin 300387, China

${ }^{2}$ School of Materials Science and Engineering, Tiangong University, Tianjin 300387, China

${ }^{3}$ Institute for Frontier Materials, Deakin University, Geelong, Victoria 3216, Australia

${ }^{4}$ Department of Chemistry and Applied Biological Science, South Dakota School of Mines and Technology, Rapid City, South Dakota 57701, United States

† These authors contributed equally to this work.

* Corresponding authors (emails: wwy-322@126.com (Wang W); Zhengtao.Zhu@sdsmt.edu (Zhu Z))
} 
to diffuse water across the hydrophobic/hydrophilic contact lines; as a result, the efficiency of solar evaporation was accelerated. Moreover, hydrophilic materials combining with hydrophobic materials could form a Janus structure to avoid salt accumulation during long-term solar desalination tests, which has been realized by previous publications [38-42]. However, structures designed for acceleration of vapor escape, which may further improve the water purification efficiency of the solar evaporator, are rarely reported.

One of the challenging issues of a solar evaporation system is that the highly humid and hot chamber of a solar still under continuous sunlight may have a detrimental effect on the system efficiency. Interestingly, a natural phenomenon has attracted our attention in the design of structures to overcome the humid environment and accelerate the vapor escape in the solar evaporator. Transpiration of a plant refers to the process that leaves release water vapor into the atmosphere. Transpiration of the plant leaves in the tropical rainforest is different from that in other regions due to their unique environment. Specifically, most of the leaves are exposed to less sunlight in dense forests, the air in a tropical rainforest has saturated vapor pressure close to the limit value because of high annual rainfall (typically higher than $2000 \mathrm{~mm}$ ), and the tropic area has high average temperatures [43]. To acclimate to the harsh environment of high humidity, high temperature, and less light, the leaves in the tropical rainforest have gradually developed a unique leaf structure, which helps maintain the metabolic balance of the plant. Scientists have studied the leaves of tropical plants and found that water evaporates quickly along the edge of the pores on the surfaces of leaves, a phenomenon known as the "edge effect" [44].

In this paper, a leaf-biomimetic structure for interfacial solar evaporator with greatly enhanced solar vapor generation efficiency is presented. The leaf-biomimetic structure, denoted as $\mathrm{HPF}$, is composed of a polypyrrole (PPy)-coated hydrophilic polyvinyl alcohol (PVA) foam covered by a micro/nano-scale porous and hydrophobic surface layer. With the addition of the porous and hydrophobic top layer, the escape of the water vapor in the leaf-biomimetic structure is accelerated. By optimizing the pore distribution of the layer, the interfacial solar evaporator achieves a rate of $3.09 \mathrm{~kg} \mathrm{~m}^{-2} \mathrm{~h}^{-1}$ with $98 \%$ efficiency under one sun irradiation $\left(1 \mathrm{~kW} \mathrm{~m}^{-2}\right)$. COMSOL simulation explains how the hydrophobic porous surface layer can modulate the diffusion behavior of water vapor at the molecular level, thereby resulting in acceleration of the evaporation process; the MATLAB Monte Carlo Simulation of the water molecule collisions in a hydrophobic pore further reveals that the water vapor molecules escape the pore faster near the wall of the pore (i.e., "edge effect"), which contributes to the high efficiency of the interfacial solar evaporator. A prototype solar water purification box based on HPF produces purified water from sea water at an average yield of $\sim 1.4 \mathrm{~L} \mathrm{~m}^{-2} \mathrm{~h}^{-1}$ under outdoor sunlight in summer, demonstrating its potential for solar desalination application.

\section{EXPERIMENTAL SECTION}

\section{Chemicals and materials}

Pyrrole and poly(styrene-co-acrylonitrile) (SAN) were procured from Sigma-Aldrich. Ferric chloride and chloroform were acquired from Fengchuan, China. PVA foam $\left(46 \mathrm{~kg} \mathrm{~m}^{-3}\right)$ was obtained from a local fabric market.

\section{Fabrication of PPy-foam (PF)}

The top part of the commercial PVA foam $(6 \mathrm{~cm}$ in diameter $)$ was immersed in $5 \mathrm{~mL}$ ferric chloride solution $(0.05,0.10$, or 0.15 mol ferric chloride in $100 \mathrm{~mL}$ ethanol). Then, the impregnated PVA foam was placed in a vacuum chamber with liquid pyrrole $(2 \mathrm{~mL})$ for $6 \mathrm{~h}$. Finally, the PPy-coated PVA foam was taken out of the chamber and the redundant iron ions were flushed out with ethanol several times, followed by drying in a vacuum oven for $5 \mathrm{~h}$ at $65^{\circ} \mathrm{C}$. The PFs prepared with different concentrations of ferric chloride solutions $(0.5,1$, and $\left.1.5 \mathrm{~mol} \mathrm{~L}^{-1}\right)$, were expressed as PF-1, PF-2 and PF-3, respectively.

\section{Self-assembly of honeycomb structured porous layer}

$\mathrm{SAN}$ in $\mathrm{CHCl}_{3}$ solution $(0.016,0.018,0.020,0.022$ and $0.024 \mathrm{~g} \mathrm{~mL}^{-1}$ ) was prepared by stirring the $\mathrm{SAN} / \mathrm{CHCl}_{3}$ solution at ambient temperature for $6 \mathrm{~h}$. The SAN/ $\mathrm{CHCl}_{3}$ solution $(2 \mathrm{~mL})$ was dispersed on an ethanol-cleaned glass substrate. The substrate was then placed in a container with an air inlet and an air outlet. Nitrogen $\left(1 \mathrm{~L} \mathrm{~min}^{-1}\right)$ was successively passed through a bottle filled with water (heated at $80^{\circ} \mathrm{C}$ ) and an empty bottle (to prevent the formation of liquid water droplets). The moist nitrogen was injected into the container through the air inlet. After $30 \mathrm{~min}$ of curing reaction, the substrate was taken out of the container and put into a vacuum oven at $65^{\circ} \mathrm{C}$ for $1 \mathrm{~h}$.

\section{Fabrication of HPF}

The HPF structure was fabricated by coating the PVA foam with PPy followed by hot pressing the honeycomb structured SAN film on the surface. Table S1 lists the conditions and detailed parameters for the preparation of the HPF structures with different concentrations of $\mathrm{SAN} / \mathrm{CHCl}_{3}$ solutions will be discussed in the RESULTS AND DISCUSSION section.

\section{Materials characterizations}

Scanning electron microscopy (SEM) characterization was carried out with a Hitachi S4800 field emission SEM (FESEM). The pore diameter distribution and porosity of the samples were assessed by Automatic Mercury Porosimeter (AutoPore lv 9510). The mechanical properties were analyzed by a general material testing machine (CMT5205, MTS Systems, China). The chemical structures of the samples were characterized by Fourier transform infrared (FTIR) spectroscopy (VERTEX 70 spectrometer, iS50, Nicolet) and thermogravimetric analysis (TGA, NETZSCH). Water contact angle (WCA) was recorded and analyzed by a contact angle meter (Kruss, DSA225). The enthalpy of vaporization of water was evaluated by differential scanning calorimetry (DSC, $200 \mathrm{~F} 3 \mathrm{Maia}^{\circledR}$, NETZSCH). The optical transmittance $(T)$ and reflectance $(R)$ of the samples were chronicled by an ultraviolet-visible-near infrared spectrometer (UV-Vis-NIR, UH4150, HITACHI) outfitted with an integrating sphere. By using the equation of $A=1-R-T$, the absorptance (A) was figured out. The thermal parameters (i.e., thermal diffusivity and thermal conductivity) were measured by a thermal conductivity analyzer (Hot Disk, Sweden). The water states in the samples were investigated by a low field nuclear magnetic resonance (LNMR) instrument (NMI20-015V-I, NIUMAG). The Raman spectra of the samples were measured and recorded using a laser confocal Raman spectroscopy (XploRA PLUS, Horiba). The ion concentration was measured by inductively coupled plasma mass spectrometry (Agilent $7500 \mathrm{ce}$ ). In order to 
improve the test accuracy, the test sample was diluted in $2 \%$ nitric acid to make the loaded ion concentration below $10 \mathrm{ppm}$.

\section{Solar vapor generation}

In order to evaluate the capability of solar vapor generation, water loss in a beaker containing different water samples was measured. The HPF samples floating on water in the beaker were illuminated by a solar simulator (PLS-SXE300D, Beijing Perfectlight, China) equipped with a standard AM 1.5 G spectral filter. The temperature distribution of the surface of the HPF structure was monitored by an infrared thermal camera (FLUKE TiX520) during the experiment. A high-precision balance (ADAM, Nimbus) recorded the mass change of the beaker, which was then used to calculate the evaporation rate and solar vapor generation efficiency. The typical ambient temperature and humidity of the in-door experiment were $20^{\circ} \mathrm{C}$ and $\sim 60 \%$, respectively.

\section{RESULTS AND DISCUSSION}

\section{Leaf-inspired design of 3D structure for the HPF-based evaporator}

Leaf from a tropical rainforest displays characteristic structures of hierarchical networks and interconnected channels that pump and transport water via transpiration (Fig. 1a-c). As schematically illustrated in Fig. 1d (left), the leaf structure includes three parts with different features and functions: (1) the stomas at the surface layer of the leaf for vapor diffusion [45]; (2) the sub-
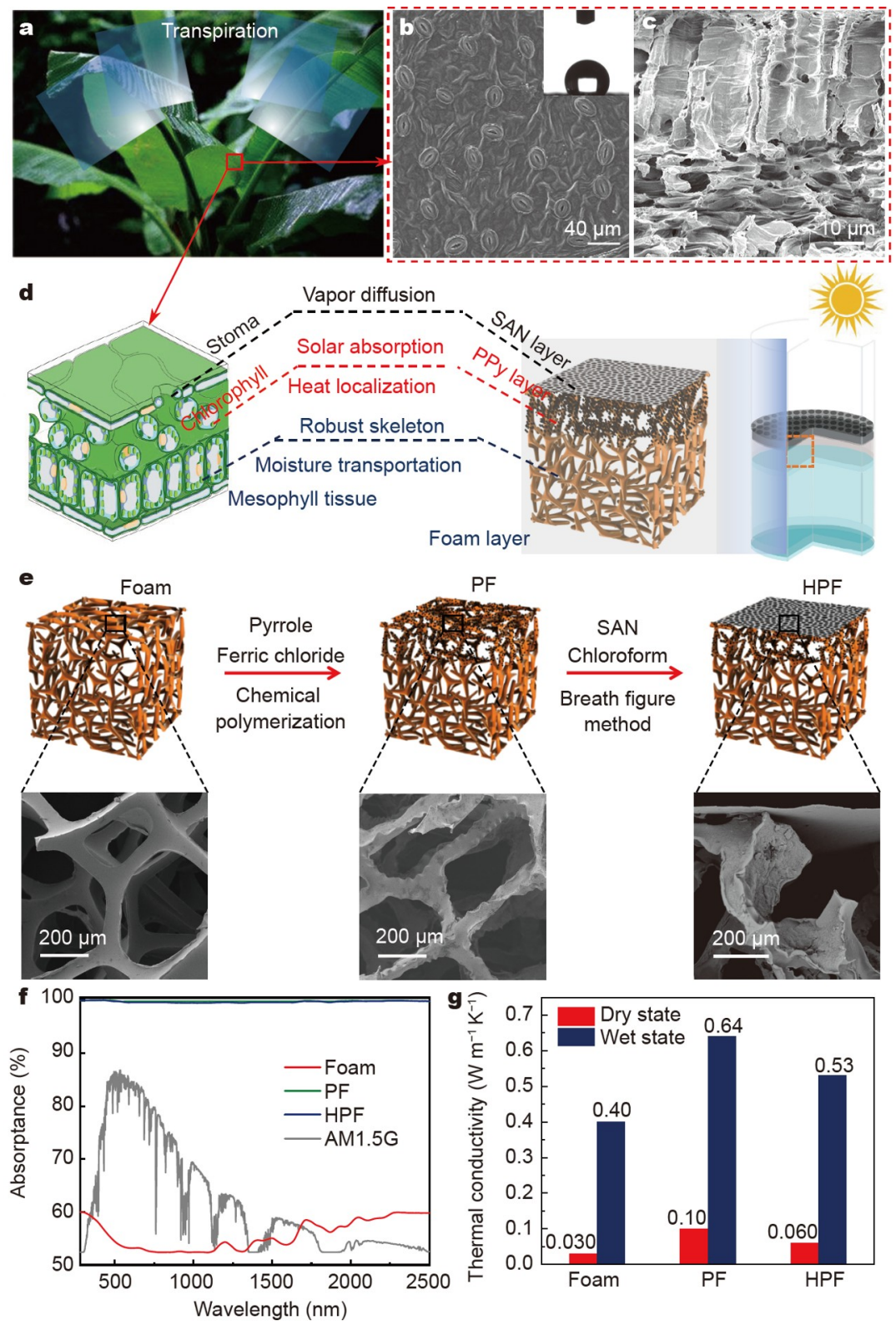

Figure 1 The biomimetic HPF structure. (a) The typical plant leaves in a tropical rainforest and (b, c) the high-magnification SEM images of the surface and the cross section of a leaf, respectively. The inset of (b) shows the WCA of the leaf surface. (d) Illustration of the structures of a leaf and the designed HPF structure for highly efficient solar vapor generation. (e) Schematic illustration of the fabrication of the biomimetic HPF structure and the corresponding SEM images of the PVA foam, PF and HPF, respectively. (f) Optical absorptance of the PVA foam, PF, and HPF. (g) Thermal conductivities of the PVA foam, PF, and HPF in the dry and wet states. 
stomal cells at the middle layer containing copious chlorophyll, which absorbs solar energy for photosynthesis and provides energy for transpiration [46]; (3) the mesophyll tissues at the bottom layer, which absorb water like a sponge from the roots and stems to provide sufficient water for evaporation. In such a unique structure, the mass of water vapor diffusing through the stomas of a plant is 50 times more than that through bulk water with the same area [47]. The high efficiency of stoma for water vapor escape can be attributed to the increased evaporation area of the substomatic cavities and the enhanced vapor diffusion by stomas. During the evaporation process, the number of water molecules evaporated near the edge of a stoma is larger than that near the center of the stoma; such phenomenon is called the "edge effect", proposed by Stefan [44] in 1882. The "edge effect" is related to the leaf surface morphology, the stomal number, and the evaporation area [48].

Inspired by the high efficiency of solar vapor transpiration of a leaf, we have designed a 3D material structure, denoted as HPF, which mimics the structure of a leaf for efficient solar vapor generation, as shown in Fig. 1d (right). The fabrication process of the HPF structure is shown in Fig. 1e, and the experimental details can be found in the EXPERIMENTAL SECTION.

The leaf-inspired HPF structure has the following components: (1) the foam layer (equivalence to the mesophyll tissue layer in a leaf) with hydrophilicity, high porosity, and low thermal conductivity as the skeleton of the solar evaporator. The foam layer provides excellent mechanical support and encourages the rapid water transport to refill the water content of the top evaporation layer. Here, the commercially available PVA foam was adopted as the framework for solar evaporator, attributing to its mechanical endurance (Fig. S1), hydrophilicity (Fig. S2), and high porosity (Fig. S3). The PVA foam has an average diameter of $196 \mu \mathrm{m}$ and the porosity of $48 \%$, indicating plenty of interconnected open channels, which can facilitate water pumping and vapor exhaling. (2) The middle layer of the photothermal PPy (equivalence to the chlorophyll layer in a leaf) coated on the PVA foam. PPy was coated on the upper side of the PVA foam by chemical polymerization to construct a bilayer foam. The PPy layer as an excellent solar absorber would assimilate and convert solar energy into internal energy to heat water confined in the porous foam network, achieving heat localization in the upper layer of the HPF structure. Fig. S4 shows that the white foam becomes black after being coated with the PPy layer, indicating strong absorptance of the visible light. Moreover, the coating of the PPy layer in the HPF structure was optimized in terms of broadband absorption, morphology, porosity, and mechanical properties, as shown in Figs S5-S8. (3) The SAN layer (equivalence to the stomal layer in a leaf). SAN was used to assemble a micro/nano-scale porous hydrophobic surface layer (Fig. S9) on the top of the HPF structure by the breath figure method, a self-assembly method for forming ordered pattern arrays based on the evaporative cooling and subsequent water droplet template $[49,50]$. The SAN hydrophobic layer had a similar function to the stomas in a leaf for accelerating water vapor escape, which would result in high vapor diffusion and efficient solar vapor generation. The stressstrain curves of the leaf-mimic structures with the SAN layer prepared under different conditions all showed mechanical properties similar to that of the polymeric PVA foam (Fig. S10), suggesting that the SAN hydrophobic layer was mostly formed on the upper surface rather than the entire polymeric network of the foam.

\section{Characterizations of the HPF structure}

The chemical composition of the HPF structure was characterized by FTIR spectroscopy, as shown in Fig. S11a. Characteristic vibrational modes of PVA $\left(1082 \mathrm{~cm}^{-1}\right.$, corresponding to the C-O stretching) [27], PPy (1530 and $1040 \mathrm{~cm}^{-1}$, corresponding to the $\mathrm{C}=\mathrm{C}$ bonding and the $\mathrm{N}-\mathrm{H}$ bonding, respectively) [16], and SAN (1500 and $1450 \mathrm{~cm}^{-1}$, corresponding to the benzene ring, and $2240 \mathrm{~cm}^{-1}$ corresponding to the $\mathrm{C} \equiv \mathrm{N}$ bonding) [51] were observed in the IR spectra of HPF, thus confirming the successful deposition of PPy and SAN layers in the PVA foam. Additionally, XPS results further prove the PPy and SAN were coated on the PVA foam (Fig. S11c). The TGA curves of the PVA foam, the PPy powders, PF, SAN resins, and HPF (Fig. S11b) showed that the HPF structure had good thermal stability up to about $250^{\circ} \mathrm{C}$ with minimum weight loss.

The solar absorption properties of the PVA foam, PF, and HPF were evaluated by UV-Vis-NIR spectroscopy. Benefiting from the intrinsic broadband absorption of PPy and the multiscattering of light in the $3 \mathrm{D}$ network structure, PF and HPF showed excellent absorptance in the entire solar spectral range (Fig. 1f). The average absorptance of HPF from 400 to $2500 \mathrm{~nm}$ was $\sim 99 \%$, indicating HPF could capture the incident solar light efficiently. In addition, because of the low density $\left(\sim 46 \mathrm{~kg} \mathrm{~m}^{-3}\right)$ of the PVA foam, HPF could self-float on water (Fig. S12). The thermal insulation performance of the HPF structure was investigated by a thermal conductivity analyzer. As shown in Fig. 1g, the PVA foam had an extraordinarily low thermal conductivity $\left(0.03 \mathrm{~W} \mathrm{~m}^{-1} \mathrm{~K}^{-1}\right)$. Notably, after coating of the PPy and SAN layers, both PF and HPF maintained a low thermal conductivity similar to the PVA foam. Even at the wet state, PF and HPF had low thermal conductivities of 0.64 and $0.53 \mathrm{~W} \mathrm{~m}^{-1} \mathrm{~K}^{-1}$, respectively. The self-floating and low thermal conductivity ensured that the thermal energy was limited in the HPF structure rather than being lost to the bulk water. Additionally, the finite thickness of the PPy layer would confine the converted thermal energy at the liquid-vapor generation interface, resulting in strong heat localization. (Figs S12 and S13).

\section{Performance of solar vapor generation under one sun}

The stomas of a leaf act as channels of gas exchange between a plant and the ambience. The transpiration rate is controlled by the size and size distribution of stomas [52]. The hydrophobic and porous SAN layer mimics the function of stomas of a leaf. In order to investigate the effects of the SAN layer of HPF on the solar vapor evaporation process, the SAN layer was prepared with various pore sizes and size distributions by controlling the concentration of the SAN solution during the preparation process. As shown in Fig. 2a-e, with an increase of the polymer solution concentration from HPF-1 to HPF-5, the porous morphology of the SAN layer was maintained while the pore size decreased. The average pore size and the total perimeter per $100 \mu \mathrm{m} \times 100 \mu \mathrm{m}$ were calculated from the SEM images, and the results are shown in Fig. $2 \mathrm{f}$. The average pore diameters of HPF1 to HPF-5 were 8.4, 7.9, 3.0, 2.7 and $4.4 \mu \mathrm{m}$, respectively. Meanwhile, the total pore perimeters per $100 \mu \mathrm{m} \times 100 \mu \mathrm{m}$ were $2104.8,3439.9,4615.6,2411.4$ and $1151.7 \mu \mathrm{m}$ for HPF-1 to HPF5 , respectively (see details in Fig. S14 and Table S1). The result could be explained by Henry's theory [53]. In a solution with high concentration, the solvent has a low vapor pressure and the 

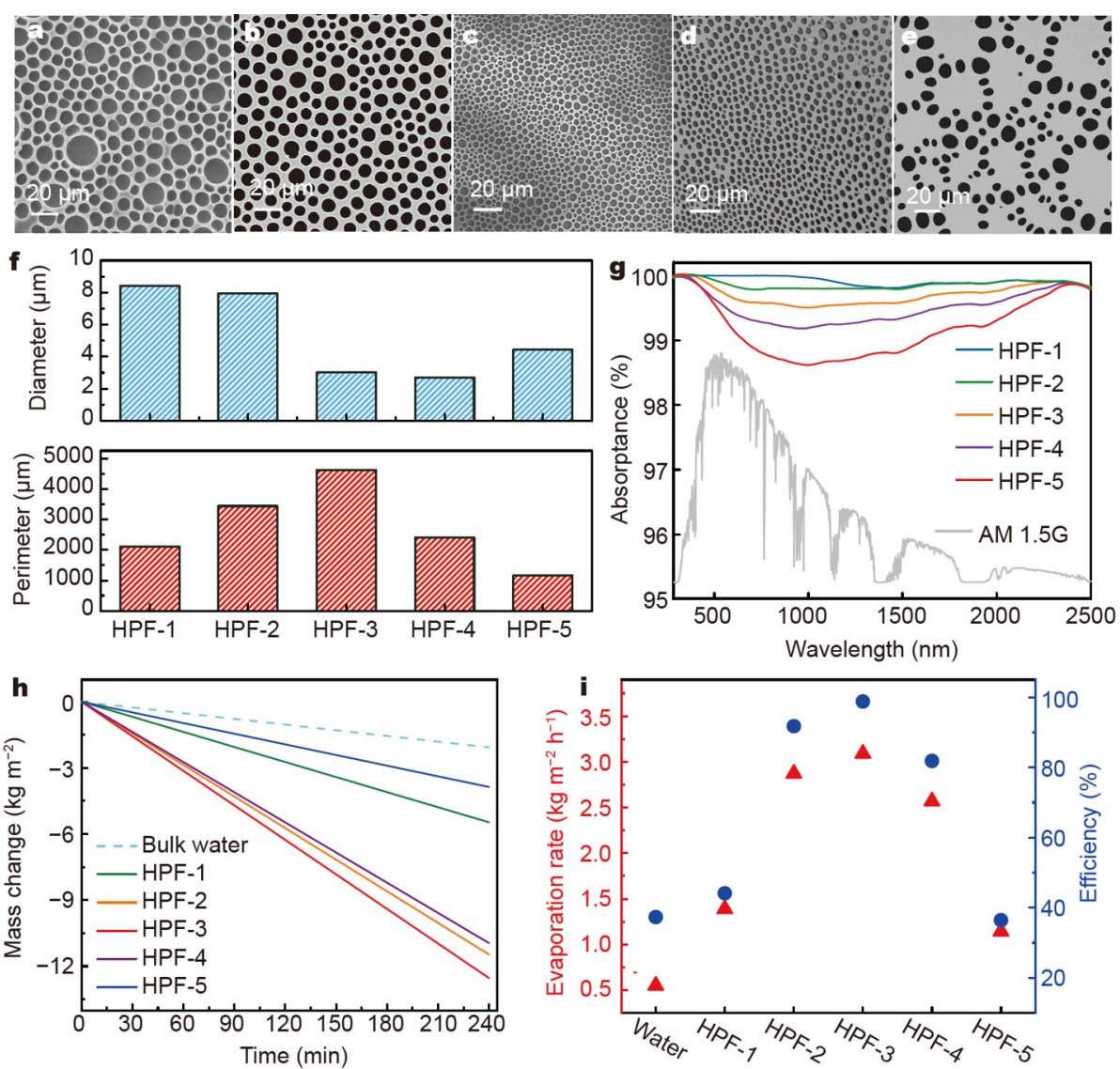

Figure 2 Performances of the leaf-mimic HPF structure for solar vapor generation. (a-e) SEM images of the surface of the HPF structures prepared with different SAN concentrations (denoted as HPF-1, HPF-2, HPF-3, HPF-4, and HPF-5, respectively). (f) Average pore size and the equivalent perimeter per $100 \mu \mathrm{m} \times 100 \mu \mathrm{m}$ of the HPF structures. (g) Solar absorptance of the HPF structures. (h) Mass change of water under 1 sun irradiation in a solar evaporator containing HPF floating on water. (i) Evaporation rate and solar thermal conversion efficiency of the solar evaporator based on the HPF structures. "Water" refers to the bulk water without the HPF structure.

solution surface temperature increases, so the water droplets condense and grow slowly, which results in a small pore size and high total perimeter per area in the film. However, when the concentration is too high, the polymer solution becomes so viscous that the water droplets are hard to immerse in the solution during the preparation process, resulting in decreased number of pores as well as the decreased total perimeter per unit area in the SAN film.

The different SAN layers had little effect on the broadband absorptance of the HPF structures. As shown in Fig. 2g, all samples had absorptance more than $98 \%$ over the solar spectrum (wavelength from 400 to $2500 \mathrm{~nm}$ ) with negligible optical loss. On the other hand, the SAN layer of the HPF structure had significant effects on the water evaporation rate when the HPF solar evaporator was operated under simulated solar irradiation. Fig. S12 shows the experimental setup of solar evaporator. Fig. $2 \mathrm{~h}$ presents the mass change of water under simulated solar irradiation $\left(1 \mathrm{~kW} \mathrm{~m}^{-2}\right)$ with and without the HPF solar evaporator floating on water. The HPF-3-based solar evaporator had a remarkably high evaporation rate of $3.09 \mathrm{~kg} \mathrm{~m}^{-2} \mathrm{~h}^{-1}$, compared with the evaporation rate of $1.38 \mathrm{~kg} \mathrm{~m}^{-2} \mathrm{~h}^{-1}$ for HPF$1,2.87 \mathrm{~kg} \mathrm{~m}^{-2} \mathrm{~h}^{-1}$ for HPF-2, $2.56 \mathrm{~kg} \mathrm{~m}^{-2} \mathrm{~h}^{-1}$ for HPF-4, and $1.14 \mathrm{~kg} \mathrm{~m}^{-2} \mathrm{~h}^{-1}$ for HPF-5. This result is in consistent with the mechanism discussed in the later section. The water vapor diffuses and escapes through the hydrophobic SAN layer with an accelerated speed at the edge of pores, and the large pore perimeters result in the high evaporation rate. The inferior performances of HPF- 5 and HPF- 1 can be attributed to the small value of the pore perimeter, which is a combination of the number of pores and the pore diameter in the unit area. The evaporation rates of the HPF-based solar evaporators were all markedly higher than the evaporation rate of $0.55 \mathrm{~kg} \mathrm{~m}^{-2} \mathrm{~h}^{-1}$ for bulk water under solar irradiation. Additional control experiments using the PVP foam and PF (i.e., the PPy-coated PVA foam) further demonstrated the significantly positive contribution of the porous and hydrophobic SAN layer to the water evaporation rate (Fig. S15). Fig. S16 shows the stable performance of the HPF evaporator, which demonstrates its potential for long-term application. Notably, the cost of one piece of HPF is as low as ca. $¥ 0.763$ (Table S2). We have compared the evaporation rates (under 1 sun) of this work and previous work in Fig. S17, indicating the high solar evaporation performance of HPFs.

The following equation can be used to calculate the solar thermal energy conversion efficiency $\left(\eta_{\mathrm{th}}\right)$ :

$\eta_{\mathrm{th}}=\dot{m} h_{v} / C_{\mathrm{opt}} P_{0}$,

where $\dot{m}$ is the mass change, $h_{v}$ is the enthalpy change of vaporization of the water in HPFs (Table S3), $P_{0}$ is the solar radiation power density of one sun $\left(1 \mathrm{~kW} \mathrm{~m}^{-2}\right)$, and $C_{\text {opt }}$ refers to the optic concentration on the surface of absorber (in our 
experiment, $C_{\mathrm{opt}}=1$ ). The solar thermal conversion efficiency of the HPF-based evaporator is shown in Fig. 2i. The solar thermal conversion efficiencies of HPF-1 to HPF-5 were 44\%, 91\%, 98\%, $81 \%$, and $36 \%$ under one sun, respectively. The solar thermal conversion efficiency of HPF-3-based evaporator reached $98 \%$ under one sun, indicating that HPF-3 would have the aptitude for highly efficient solar evaporation under natural sunlight. In other words, HPF-3 presented the highest evaporation rate, outperforming PF and other HPFs. The heat loss of HPF-3 was calculated to be $14.47 \%$ (Note S1 and Table S4). It is worth noting that during solar evaporation, the water temperatures under the HPF1-HPF5 increased slightly by $1.7,1.0,0.9,1.5$, and $2.2^{\circ} \mathrm{C}$, respectively, indicating HPF-3 showed excellent thermal insulation.

\section{Mechanism for high-efficiency solar evaporation based on HPF}

For high-efficiency solar evaporator based on innovative material design, researchers have been focusing on maximizing the solar absorptance, minimizing the heat loss, localizing the heat at the photothermal conversion region, and regulating water transport [54,55]. In our work, a solar evaporator with unidirectional and controllable water transportation was designed to achieve an ideal dynamic equilibrium of water replenishment and evaporation, which resulted in a solar evaporator with a water evaporation rate of $1.3 \mathrm{~kg} \mathrm{~m}^{-2} \mathrm{~h}^{-1}$, reaching $81 \%$ of the theoretical evaporation rate of $1.6 \mathrm{~kg} \mathrm{~m}^{-2} \mathrm{~h}^{-1}$ under one sun illumination. The theoretic evaporation rate, i.e., the maximum mass flux, was calculated using Equation (1) by assuming 100\% efficiency, the vaporization enthalpy of the bulk water being $2444 \mathrm{~kJ} \mathrm{~g}^{-1}, P_{0}$ being $1 \mathrm{~kW} \mathrm{~m}^{-2}$, and $C_{\text {opt }}$ being 1 . In this work, the evaporation rate of the HPF-3 solar evaporator was $3.09 \mathrm{~kg} \mathrm{~m}^{-2} \mathrm{~h}^{-1}$, much higher than the theoretic evaporation rate of $1.6 \mathrm{~kg} \mathrm{~m}^{-2} \mathrm{~h}^{-1}$. Noticeably, the theoretic evaporation rate was calculated based on the vaporization enthalpy of the bulk water, whereas water in the HPF structure was confined in the pores of the PVA foam. Additionally, the hydrophobic and porous SAN layer may accelerate the water vapor escaping rate, similar to the stomal layer in a leaf. To explore the underlying mechanism of the ultrahigh evaporation rate in the leaf-mimic HPF-based solar evaporator, various experimental techniques and theoretic simulations were used to study the states of water in the HPF structure and the edge effect of the hydrophobic and porous SAN layer.

The proposed mechanism of the high evaporation rate of the HPF-3 solar evaporator is illustrated in Fig. 3a. In PVA, the polymer chains are rich with $-\mathrm{OH}$ groups. These $-\mathrm{OH}$ groups of PVA interact with the water molecules by hydrogen bonds, which may be strong enough to weaken the hydrogen bonds among the water molecules themselves to form water clusters in the PVA network. Due to the capillary effect of the micro-scale connected channels in the PVA foam, the water clusters can overcome gravity and transport upward. The PPy layer deposited on the top of the PVA skeleton absorbs solar energy and generates heat, which provides enough thermal energy to break the hydrogen bonds of the water clusters to produce water vapor. Finally, the water vapor diffuses and escapes through the hydrophobic SAN layer with an accelerated speed at the edge of pores, resulting in the high evaporation rate.

Previous research has suggested that water in a polymer network could form water clusters with varied configurations [56]. According to the intermolecular hydrogen bond interaction, water in hydrated polymer networks can be divided into three categories: free water (FW), intermediate water (IW), and bound water. As shown in Fig. 3b, c, the $\mathrm{O}-\mathrm{H}$ stretching modes in the Raman spectra were deconvoluted to analyze the states (or configurations) of water in bulk and the HPF pores [57]. The modes at 3233 and $3401 \mathrm{~cm}^{-1}$ are assigned to $\mathrm{FW}$ formed by the interaction of two protons and two electron lone pairs of oxygen with adjoining water molecules, while the modes at 3514 and $3630 \mathrm{~cm}^{-1}$ are related to weak hydrogen-bonded IW. In the spectrum of the bulk water (Fig. $3 \mathrm{~b}$ ), the areas of the modes assigned to FW are dominating. On the other hand, in the spectrum of water in HPF (Fig. 3c), the areas of the modes of IW are comparable to those of FW, indicating that a higher proportion of IW in the HPF structure. These results confirm that the water molecules in the PVA network of the HPF structure have distinctly different states from the bulk water.

The different water states in HPF were further verified by the transverse relaxation time of the samples in the LNMR measurement [58-60]. The transverse relaxation time represents the fluidity of water. As shown in Fig. S18, the three plots from left to right represent the transverse relaxation times of bound water, IW, and FW, respectively. Evidently, the signals of IW in the PVA foam, PF and HPF were higher than that in bulk water, confirming that the water clusters with weak intermolecular interactions were formed in the PVA network. Such weak interactions of the IW clusters with the polymer chains and adjacent water molecules would lead to reduced energy barriers for water evaporation. The curves of the DSC analysis indicated that the enthalpy of evaporation of water in HPF was reduced in the PVA polymer network structure, providing additional evidence of the lower energy barriers for water evaporation in the HPF structure (Fig. S19 and Table S3). To further estimate the vaporization enthalpy of water during the solar evaporation process, we recorded the evaporation rate in dark condition and calculated the equivalent vaporization enthalpy (details in Table S5). As shown in Tables S3 and S5, the vaporization enthalpy of bulk water is much higher than that of water in HPF.

In a typical transpiration process, liquid water is transported to the mesophyll cells and then evaporated in the substomatal cavity. The saturated vapor in the cavity diffuses into the atmosphere through the stomas. The rate of diffusion is higher at the edges than that near the center of stomas, as shown in Fig. 3d; such phenomenon is called the "edge effect" in stomatal transpiration. During the process, the low probability of collision between the water molecules at the edge of a stoma results in less diffusion resistance near the edge when water vapor passes through the stoma. Notably, the evaporation rate of water during the stomal transpiration is approximately dozens of times higher than that of the free surface with the same area [61]. Based on the similarity of the HPF structure and a leaf, the edge effect may take place when water vapor molecules pass through the pores of the SAN layer in HPF. COMSOL multiphysics simulations were used to gain supplementary understanding of the edge effect in the HPF solar evaporator (see Fig. S20 and Section S6.3 for details). As shown in Fig. 3e, simulated vapor diffusion flux in a single pore with a size of $30 \mu \mathrm{m}$ is the highest at the edge of the pore, implying that the edge effect would take place in the pores of the SAN layer of HPF in the process of evaporation. The collision behavior of the water molecules in a membrane pore was further simulated using MATLAB. In the simulation, as shown in Fig. 3f, "vapor" water molecules at the bottom of a 


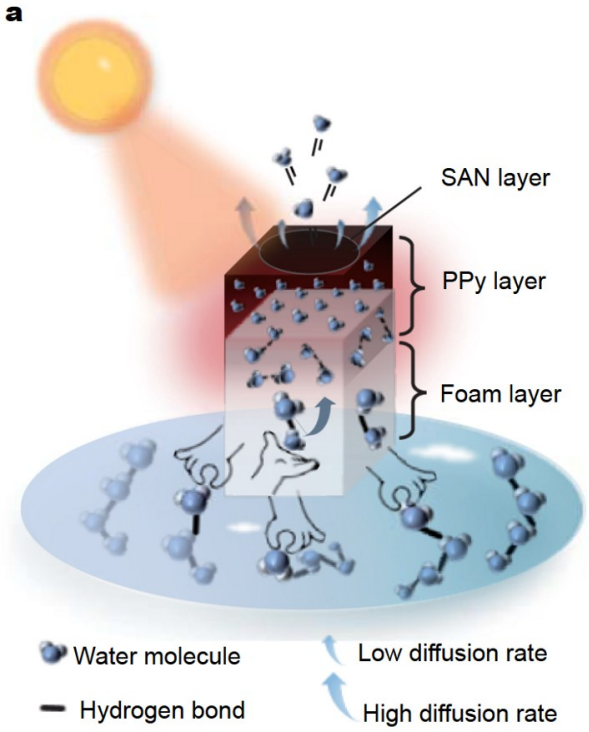

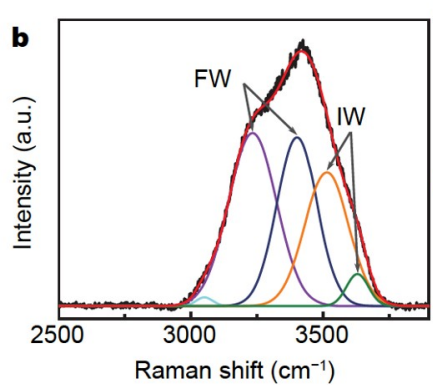

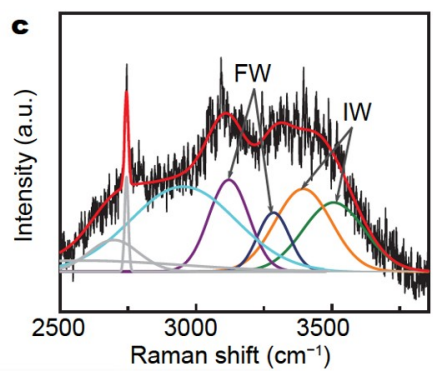

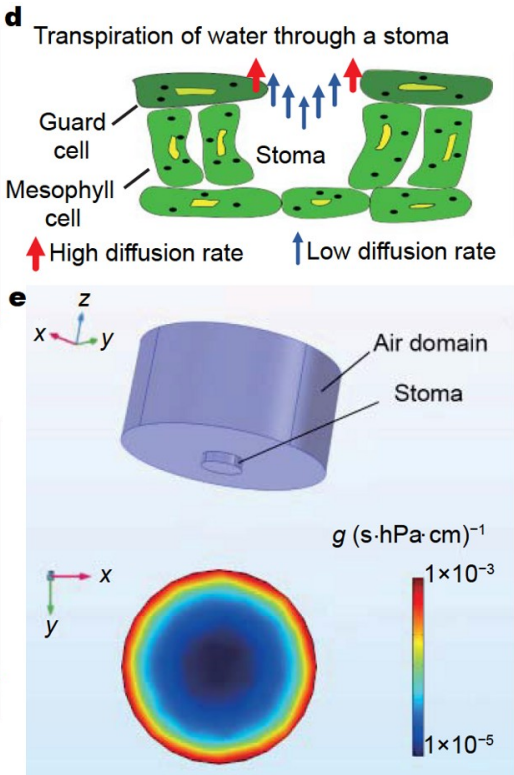

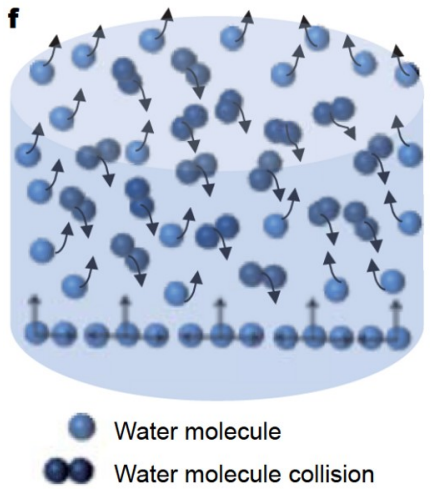

g

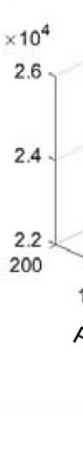

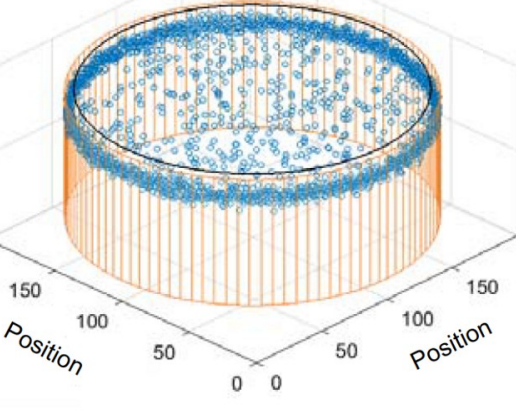

Water molecular distribution

h

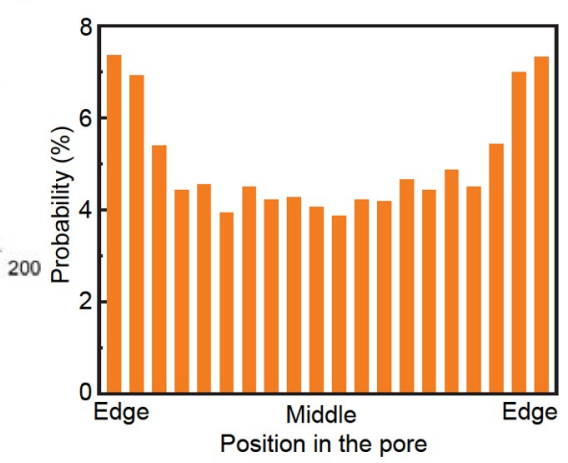

Figure 3 Schematic of the HPF-based evaporator for highly efficient and continuous solar vapor generation. (a) The proposed mechanism of the states and movement of water molecules in the HPF structure. $(b, c)$ Raman shift of the -OH group of bulk water and water confined in the HPF structure, respectively, indicating that the water clusters exist in the HPF structure. (d) The edge effect in stomal transpiration. (e) Water vapor diffusion flux in a pore of size $30 \mu \mathrm{m}$ simulated using the COMSOL multiphysics modeling. (f) Schematic diagram of water molecules in a membrane pore. The MATLAB simulation results of the distribution $(\mathrm{g})$ and distribution probability $(\mathrm{h})$ of the "vapor" water molecules that reached the top of the pore after collision and diffusion in the membrane pore, respectively.

pore escape the pore when they move randomly to the top. To simplify the computing model, during the process, the collided "vapor" water molecules "disappear," i.e., they do not escape the pore. Fig. 3g, h show the distribution and probability of the "vapor" water molecules that move up to the top of the pore after the "vapor" water molecules collide and diffuse randomly in the membrane pore. Evidently, more "vapor" molecules at the edge of the pore can move to the top and therefore escape to the open space at a higher diffusion rate, in line with the COMSOL multiphysics simulation result. Additionally, in dark, water covered with the HPF structures evaporated at a faster rate than the free surface water (i.e., bulk water) (Fig. S21), confirming the micro/nano-scale hydrophobic SAN pores on the surface of HPF accelerate water evaporation. The HPF-3 structure has the highest perimeter per unit area, and therefore, would be expected to have the most pronounced "edge effect" for accelerating water vapor escape. These results support that the optimal solar evaporation efficiency has been achieved in the HPF-3 structure.

\section{Laboratory and outdoor solar seawater desalinization}

To demonstrate the solar seawater desalinization capability of the leaf-mimic structure, the brine samples with the characteristic simulated salinities of $8 \%$, $36 \%$ and $100 \%$ (\%o represents $g$ of dissolved salt per $\mathrm{kg}$ seawater) were desalinated using the optimized HPF-3 solar evaporator. The salinities of the purified water were down to less than $0.1 \%$ (Fig. $4 \mathrm{a}$ ), approximately onetenth of the drinking water standard (1\%o salinity) recommended by the World Health Organization. Moreover, a Yellow Sea water sample was desalinated using the HPF-3 solar evaporator. The concentrations of the four major ions $\left(\mathrm{K}^{+}, \mathrm{Ca}^{2+}\right.$, $\mathrm{Na}^{+}$and $\mathrm{Mg}^{2+}$ ) in the desalinated water were reduced tremendously (Fig. 4b) and were lower than the values typically obtained through membrane distillation techniques (1-50 $\mathrm{mg} \mathrm{L}^{-1}$ ) [62].

An HPF-3-based solar vapor generation chamber was settled on the rooftop of the School of Textile Science and Engineering at Tiangong University campus on July 23, 2020 (summer). As shown in Fig. 4c, the HPF-3 structure with a diameter of $\sim 14 \mathrm{~cm}$ 

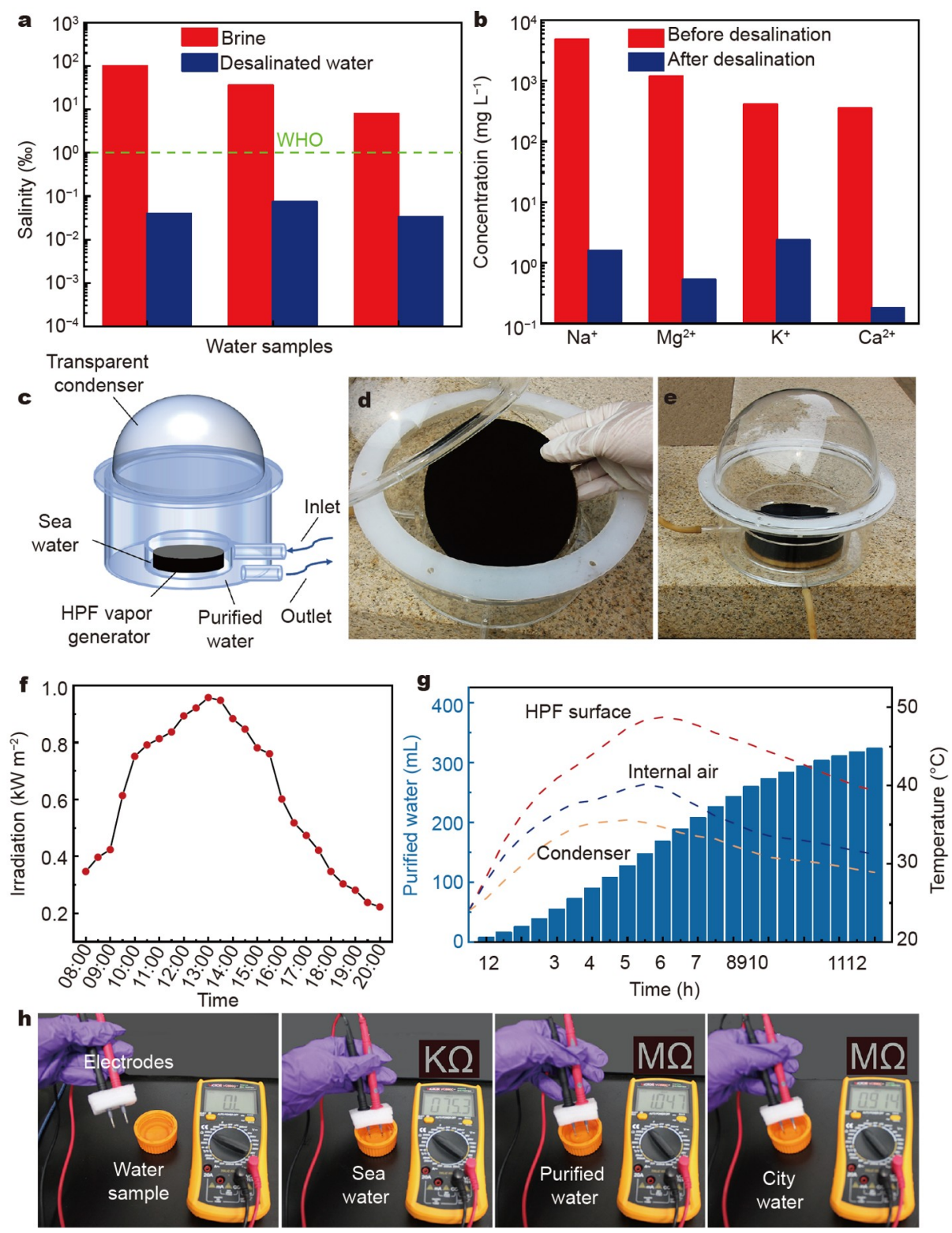

Figure 4 Solar seawater desalinization performance of the HPF-3 solar evaporator. (a) The salinities of the three simulated brine samples and the desalinated water after desalination by the HPF-3 solar evaporator. (b) Concentrations of the four major ions in the Yellow Sea water sample and the water desalinated by the HPF-3 solar evaporator. (c-e) Diagram of a water desalination device, digital photograph of a large-scale HPF structure, and a photo of the prototype solar desalination device, respectively. (f) Solar radiation intensity monitored by time on a sunny day from 8:00-20:00 on Jul. 23, 2020. (g) The mass of purified water and temperatures of the HPF surface, the internal air, and the top condenser during the day of the experiment. (h) Evaluation of the quality of the water desalination by surveying the resistance of the seawater, desalinated water, and city tap water using a multimeter with a constant distance between two electrodes.

and thickness of $2 \mathrm{~cm}$ was floating on brine in a container located at the center of the purification chamber (Fig. 4d). The steam condensed on the hyaline acrylic condenser, and the water condensate flowed to the bottom of the chamber (Fig. 4d). For continuous water purification, the water input and output of the device were connected to the brine bottle and the purified water collecting bottle, respectively (Fig. 4d, e); this set-up represented a prototype of a fully functional solar water purification equipment. The water purification was accomplished under natural sunlight with average solar irradiation of $\sim 0.6 \mathrm{~kW} \mathrm{~m}^{-2}$ for $12 \mathrm{~h}$. The solar irradiation (Fig. 4f), the air temperature, the condenser temperature, and the apparent-surface temperature of HPF-3 were cautiously tracked to quantitatively assess the utilization efficiency of the natural solar power. As shown in Fig. 4g, the apparent-surface temperature of the HPF structure rose to $\sim 50^{\circ} \mathrm{C}$, suggesting an inferior evaporation cooling performance in comparison with the bench-scale laboratory test condition. This phenomenon could be attributed to the saturated internal humidity of the closed system, which likely limited the evaporation of water. The condenser kept up a relatively low temperature of $\sim 34^{\circ} \mathrm{C}$, while the internal air heated up to $\sim 40^{\circ} \mathrm{C}$, revealing a sustainable evaporation and condensation effect in the device. With this prototype setup, an average water purification rate of $\sim 1.4 \mathrm{~L} \mathrm{~m}^{-2} \mathrm{~h}^{-1}$ (Fig. $4 \mathrm{~g}$, blue histogram) was 
achieved for the seawater under a sufficient sunny day (24$32^{\circ} \mathrm{C}$ ). The water quality can be evaluated by measuring the resistance of water between two electrodes at a constant distance (Fig. 4h). Moreover, to demonstrate the stable operation and cyclic salt-rejection capability, the HPF-3 evaporator was used to desalinate the $10 \mathrm{wt} \%$ brine water outdoor for $10 \mathrm{~h}$, and the purified water was collected and evaluated for 10 cycles. As shown in Fig. S22, during the desalination, the salinity of the purified water and the evaporation rate was steady at $0.04 \%$ and $1.4 \mathrm{~L} \mathrm{~m}^{-2} \mathrm{~h}^{-1}$, respectively. The steady evaporation rate and the salinity of purified water indicated that the HPF-3 evaporator had excellent and durable salt-rejection capability. The seawater (from the Yellow Sea), the purified water, and the domestic tap water (from a municipal water system of Tianjin, China) showed the resistance values of $75.3 \mathrm{k} \Omega, 1.047 \mathrm{M} \Omega$, and $0.914 \mathrm{M} \Omega$, respectively, revealing effective seawater desalinization by the prototype solar evaporator.

\section{CONCLUSIONS}

A leaf-mimic material structure HPF composed of the PVA foam, PPy photothermal layer, and a hydrophobic and porous SAN layer was designed for solar vapor generation. Highly efficient solar water desalination using the HPF structure was achieved as the results of strong solar absorptance and heat conversion by PPy, reduced enthalpy of vaporization of water clusters in the HPF channels, and acceleration of vapor diffusion in the pores of the SAN layer. The PVA skeleton of the HPF structure transported water expeditiously from the bulk water. The interactions between the - $\mathrm{OH}$ groups of PVA with the water molecules weakened hydrogen bonding between water molecules and water clusters, resulting in low vaporization enthalpy of water in the PVA polymeric network. The vapor diffusion was regulated by the hydrophobic and porous SAN layer on the surface of the HPF structure, providing accelerated escape of water vapor in the PPy-coated polymeric network. These unique properties of the HPF structure enabled a solar vapor generation rate of $3.09 \mathrm{~kg} \mathrm{~m}^{-2} \mathrm{~h}^{-1}$ with a solar-thermal conversion efficiency of up to $98 \%$. A prototype HPF-based solar desalination system produced desalinated water with an average yield of $1.4 \mathrm{~L} \mathrm{~m}^{-2} \mathrm{~h}^{-1}$ under natural sunlight. It can be envisioned that the HPF-based solar vapor generation system, capable of efficient and effective desalination of water, will have straight applications not only in saline/seawater treatment but also in other fields, such as sea-salt production and resource recovery.

\section{Received 30 August 2021; accepted 21 October 2021;} published online 20 December 2021

1 Mekonnen MM, Hoekstra AY. Four billion people facing severe water scarcity. Sci Adv, 2016, 2: e1500323

2 Gao M, Zhu L, Peh CK, et al. Solar absorber material and system designs for photothermal water vaporization towards clean water and energy production. Energy Environ Sci, 2019, 12: 841-864

3 Jin X, Li Y, Li W, et al. Nanomaterial design for efficient solar-driven steam generation. ACS Appl Energy Mater, 2019, 2: 6112-6126

4 Zhang $\mathrm{P}, \mathrm{Xu} \mathrm{Q}$, Liao $\mathrm{Q}$, et al. Interface-enhanced distillation beyond tradition based on well-arranged graphene membrane. Sci China Mater, 2020, 63: 1948-1956

5 Zhu HW, Ge J, Zhao HY, et al. Sponge-templating synthesis of sandwich-like reduced graphene oxide nanoplates with confined gold nanoparticles and their enhanced stability for solar evaporation. Sci China Mater, 2020, 63: 1957-1965
6 Hao L, Liu N, Niu R, et al. High-performance salt-resistant solar interfacial evaporation by flexible robust porous carbon/pulp fiber membrane. Sci China Mater, 2022, 65: 201-212

7 Liu N, Hao L, Zhang B, et al. Rational design of high-performance bilayer solar evaporator by using waste polyester-derived porous carbon-coated wood. Energy Environ Mater, 2021, eem2.12199

8 Zhang Y, Ravi SK, Yang L, et al. Portable trilayer photothermal structure for hybrid energy harvesting and synergic water purification. ACS Appl Mater Interfaces, 2019, 11: 38674-38682

9 Wang W, Aleid S, Shi Y, et al. Integrated solar-driven PV cooling and seawater desalination with zero liquid discharge. Joule, 2021, 5: 18731887

10 Wang W, Shi Y, Zhang C, et al. Simultaneous production of fresh water and electricity via multistage solar photovoltaic membrane distillation. Nat Commun, 2019, 10: 3012

11 Tao P, Ni G, Song C, et al. Solar-driven interfacial evaporation. Nat Energy, 2018, 3: 1031-1041

12 Chen S, Sun Z, Xiang W, et al. Plasmonic wooden flower for highly efficient solar vapor generation. Nano Energy, 2020, 76: 104998

13 Li Y, Jin X, Zheng Y, et al. Tunable water delivery in carbon-coated fabrics for high-efficiency solar vapor generation. ACS Appl Mater Interfaces, 2019, 11: 46938-46946

14 Yang J, Pang Y, Huang W, et al. Functionalized graphene enables highly efficient solar thermal steam generation. ACS Nano, 2017, 11: 5510-5518

15 Yin Z, Wang H, Jian M, et al. Extremely black vertically aligned carbon nanotube arrays for solar steam generation. ACS Appl Mater Interfaces, 2017, 9: 28596-28603

16 Li C, Jiang D, Huo B, et al. Scalable and robust bilayer polymer foams for highly efficient and stable solar desalination. Nano Energy, 2019, 60: 841-849

$17 \mathrm{Xu} \mathrm{W}, \mathrm{Hu}$ X, Zhuang S, et al. Flexible and salt resistant Janus absorbers by electrospinning for stable and efficient solar desalination. Adv Energy Mater, 2018, 8: 1702884

18 Liu Z, Song H, Ji D, et al. Extremely cost-effective and efficient solar vapor generation under nonconcentrated illumination using thermally isolated black paper. Glob Challenges, 2017, 1: 1600003

19 Li H, He Y, Hu Y, et al. Commercially available activated carbon fiber felt enables efficient solar steam generation. ACS Appl Mater Interfaces, 2018, 10: 9362-9368

20 Wang F, Mu P, Zhang Z, et al. Reduced graphene oxide coated hollow polyester fibers for efficient solar steam generation. Energy Technol, 2019, 7: 1900265

$21 \mathrm{Li} \mathrm{X}, \mathrm{Li} \mathrm{J}, \mathrm{Lu} \mathrm{J}$, et al. Enhancement of interfacial solar vapor generation by environmental energy. Joule, 2018, 2: 1331-1338

$22 \mathrm{Wu} \mathrm{X}, \mathrm{Wu} \mathrm{Z}$, Wang Y, et al. All-cold evaporation under one sun with zero energy loss by using a heatsink inspired solar evaporator. Adv Sci, 2021, 8: 2002501

23 Wang Y, Wu X, Yang X, et al. Reversing heat conduction loss: Extracting energy from bulk water to enhance solar steam generation. Nano Energy, 2020, 78: 105269

24 Shao B, Wu X, Wang Y, et al. A general method for selectively coating photothermal materials on $3 \mathrm{D}$ porous substrate surfaces towards costeffective and highly efficient solar steam generation. J Mater Chem A, 2020, 8: 24703-24709

25 Wang $\mathrm{Y}, \mathrm{Wu} \mathrm{X}$, Shao B, et al. Boosting solar steam generation by structure enhanced energy management. Sci Bull, 2020, 65: 1380-1388

$26 \mathrm{Mu} \mathrm{P}$, Bai W, Fan Y, et al. Conductive hollow kapok fiber-PPy monolithic aerogels with excellent mechanical robustness for efficient solar steam generation. J Mater Chem A, 2019, 7: 9673-9679

27 Zhao F, Zhou X, Shi Y, et al. Highly efficient solar vapour generation via hierarchically nanostructured gels. Nat Nanotech, 2018, 13: 489-495

28 Qiu P, Liu F, Xu C, et al. Porous three-dimensional carbon foams with interconnected microchannels for high-efficiency solar-to-vapor conversion and desalination. J Mater Chem A, 2019, 7: 13036-13042

29 Liu S, Huang C, Huang Q, et al. A new carbon-black/cellulose-sponge system with water supplied by injection for enhancing solar vapor generation. J Mater Chem A, 2019, 7: 17954-17965

30 Ghafurian MM, Niazmand H, Ebrahimnia-Bajestan E, et al. Wood 
surface treatment techniques for enhanced solar steam generation. Renew Energy, 2020, 146: 2308-2315

31 Zhang $\mathrm{H}, \mathrm{Li} \mathrm{L}$, Jiang B, et al. Highly thermally insulated and superhydrophilic corn straw for efficient solar vapor generation. ACS Appl Mater Interfaces, 2020, 12: 16503-16511

$32 \mathrm{Xu} \mathrm{N}, \mathrm{Hu} \mathrm{X}, \mathrm{Xu} \mathrm{W}$, et al. Mushrooms as efficient solar steam-generation devices. Adv Mater, 2017, 29: 1606762

33 Zhou Y, Ding T, Gao M, et al. Controlled heterogeneous water distribution and evaporation towards enhanced photothermal waterelectricity-hydrogen production. Nano Energy, 2020, 77: 105102

34 Meng FL, Gao M, Ding T, et al. Modular deformable steam electricity cogeneration system with photothermal, water, and electrochemical tunable multilayers. Adv Funct Mater, 2020, 30: 2002867

35 Yang M, Tan CF, Lu W, et al. Spectrum tailored defective 2D semiconductor nanosheets aerogel for full-spectrum-driven photothermal water evaporation and photochemical degradation. Adv Funct Mater, 2020, 30: 2004460

36 Yang $\mathrm{Y}$, Zhao $\mathrm{H}$, Yin Z, et al. A general salt-resistant hydrophilic/ hydrophobic nanoporous double layer design for efficient and stable solar water evaporation distillation. Mater Horiz, 2018, 5: 1143-1150

37 Guo Y, Zhao X, Zhao F, et al. Tailoring surface wetting states for ultrafast solar-driven water evaporation. Energy Environ Sci, 2020, 13: 2087-2095

38 Shao Y, Tang J, Li N, et al. Designing a bioinspired synthetic tree by unidirectional freezing for simultaneous solar steam generation and salt collection. EcoMat, 2020, 2: e12018

39 Liu Z, Zhou Z, Wu N, et al. Hierarchical photothermal fabrics with low evaporation enthalpy as heliotropic evaporators for efficient, continuous, salt-free desalination. ACS Nano, 2021, 15: 13007-13018

40 Zhang Y, Zhang $\mathrm{H}$, Xiong $\mathrm{T}$, et al. Manipulating unidirectional fluid transportation to drive sustainable solar water extraction and brinedrenching induced energy generation. Energy Environ Sci, 2020, 13: 4891-4902

41 Wang W, Shi Y, Zhang C, et al. Solar seawater distillation by flexible and fully passive multistage membrane distillation. Nano Lett, 2021, 21: 5068-5074

42 Zhang Y, Xiong T, Suresh L, et al. Guaranteeing complete salt rejection by channeling saline water through fluidic photothermal structure toward synergistic zero energy clean water production and in situ energy generation. ACS Energy Lett, 2020, 5: 3397-3404

43 Godderis Y, Roelandt C, Schott J, et al. Towards an integrated model of weathering, climate, and biospheric processes. Rev Mineral Geochem, 2009, 70: 411-434

44 Stefan J. Ueber die verdampfung aus einem kreisförmig oder elliptisch begrenzten becken. Ann Phys, 1882, 253: 550-560

45 Mott KA, O'leary JW. Stomatal behavior and $\mathrm{CO}_{2}$ exchange characteristics in amphistomatous leaves. Plant Physiol, 1984, 74: 47-51

46 Krause GH, Weis E. Chlorophyll fluorescence and photosynthesis: The basics. Annu Rev Plant Physiol Plant Mol Biol, 1991, 42: 313-349

47 Lack AJ, Evans DE. Instant Notes in Plant Biology ( $2^{\text {nd }}$ Ed. $)$. Oxford: Taylor and Francis, 2021

48 Kumar N, Arakeri JH. Experimental and numerical investigation of evaporation from line sources of water in low porosity surfaces. J Hydrol, 2019, 569: 795-808

49 Bunz UHF. Breath figures as a dynamic templating method for polymers and nanomaterials. Adv Mater, 2006, 18: 973-989

50 Zhao B, Li C, Lu Y, et al. Formation of ordered macroporous membranes from random copolymers by the breath figure method. Polymer, 2005, 46: 9508-9513

51 Das G, Banerjee AN. Fourier transform infrared spectroscopic studies of the poly(styrene-co-acrylonitrile) and poly(vinyl chloride-co-vinyl acetate) blends. J Appl Polym Sci, 1997, 63: 991-1000

52 Parlange JY, Waggoner PE. Stomatal dimensions and resistance to diffusion. Plant Physiol, 1970, 46: 337-342

53 Yu C, Zhai J, Gao X, et al. Water-assisted fabrication of polyaniline honeycomb structure film. J Phys Chem B, 2004, 108: 4586-4589

54 Song C, Hao L, Zhang B, et al. High-performance solar vapor generation of $\mathrm{Ni} /$ carbon nanomaterials by controlled carbonization of waste polypropylene. Sci China Mater, 2020, 63: 779-793
$55 \mathrm{Xu} \mathrm{N}, \mathrm{Li}$ J, Wang Y, et al. A water lily-inspired hierarchical design for stable and efficient solar evaporation of high-salinity brine. Sci Adv, 2019, 5: eaaw7013

56 Zhou X, Zhao F, Guo Y, et al. Architecting highly hydratable polymer networks to tune the water state for solar water purification. Sci Adv, 2019, 5: eaaw5484

57 Kudo K, Ishida J, Syuu G, et al. Structural changes of water in poly (vinyl alcohol) hydrogel during dehydration. J Chem Phys, 2014, 140: 044909

58 Zhang $\mathrm{Y}$, Wang C. Micropatterning of proteins on 3D porous polymer film fabricated by using the Breath-Figure method. Adv Mater, 2007, 19: 913-916

59 Sánchez-Alonso I, Moreno P, Careche M. Low field nuclear magnetic resonance (LF-NMR) relaxometry in hake (Merluccius merluccius, L.) muscle after different freezing and storage conditions. Food Chem, 2014, 153: 250-257

60 Sun Q, Zhang M, Mujumdar AS, et al. Combined LF-NMR and artificial intelligence for continuous real-time monitoring of carrot in microwave vacuum drying. Food Bioprocess Technol, 2019, 12: 551562

61 Aminzadeh M, Or D. Pore-scale study of thermal fields during evaporation from drying porous surfaces. Int J Heat Mass Transfer, 2017, 104: 1189-1201

62 Wae AbdulKadir WAF, Ahmad AL, Seng OB, et al. Biomimetic hydrophobic membrane: A review of anti-wetting properties as a potential factor in membrane development for membrane distillation (MD). J Industrial Eng Chem, 2020, 91: 15-36

Acknowledgements This work was supported by the Key Project of the Natural Science Foundation of Tianjin (20JCZDJC00350), the National Natural Science Foundation of China (51803108), and Tianjin Research Innovation Project for Postgraduate Students (2020YJSB066).

Author contributions $\mathrm{Li} \mathrm{Y}$ and $\mathrm{Li} \mathrm{W}$ conducted the experiment; Han $\mathrm{X}$ and Wang W performed the DSC measurement; Yang X and Niu J performed the MATLAB simulation; Lin $\mathrm{T}$ and Zhu Z performed some data analysis and offered helpful suggestions; Li $\mathrm{Y}$ and Jin $\mathrm{X}$ designed this study, analyzed the data and wrote the paper. All authors contributed to the general discussion.

Conflict of interest The authors declare that they have no conflict of interest.

Supplementary information Experimental details and supporting data are available in the online version of the paper.

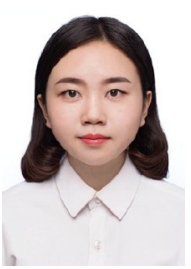

Yeran Li is currently a $\mathrm{PhD}$ candidate at the School of Textile Science and Engineering, Tiangong University. Her research mainly focuses on solar vapor generation by heat localization.

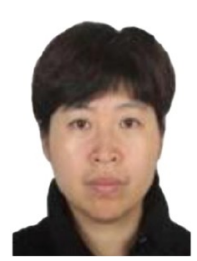

Xin Jin is a Professor at Tiangong University. She received her $\mathrm{PhD}$ degree in textile engineering from the same university in 2006. Her research activities focus on the preparation and characterization of functional fibers for energy applications. 


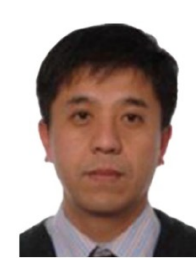

Wenyu Wang is an Associate Professor at Tiangong University, China. He received his $\mathrm{PhD}$ degree in textile engineering from Tiangong University in 2006. He worked as a $\mathrm{R} \& \mathrm{D}$ Department Manager for the Best Chance Medical Instrument Company for three years and then joined Tiangong University. His research focuses on biomaterial fibers and functional fibers for energy applications.

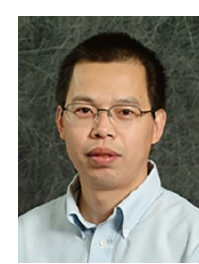

Zhengtao Zhu received his BS degree in materials chemistry and MS degree in polymer chemistry and physics from Fudan University in 1992 and 1995, respectively. He received his $\mathrm{PhD}$ degree in materials chemistry from the State University of New York at Binghamton in 2001. Currently, he is an Associate Professor at the South Dakota School of Mines and Technology. His research interests are about "nano-scaled materials and devices".
仿生微/纳米疏水多孔膜负载亲水海绵用于高效太阳 能蒸汽转化

李叶燃 ${ }^{1 \dagger}$, 金欣 $^{2 \dagger}$, 李伟 ${ }^{1}$, 牛家嵘 $^{1}$, 韩幸 $^{1}$, 杨晓芳 $^{1}$, 王闻宇 $1^{*}$, 林童 ${ }^{1,3}$, 朱正涛 $1,4^{*}$

摘要 利用光热材料吸收太阳能产生局域化的热效应实现太阳能蒸汽 转化是一项环境友好型海水淡化和废水净化的技术. 本文受自然界中 叶片蒸腾作用的启发, 设计了一种新型仿生叶片结构, 以实现高效的太 阳能蒸汽转化. 这种新型仿生叶片结构由三个部分组成: (1) 亲水性聚 乙烯醇(PVA)海绵层(相当于叶子中的叶肉组织层), 具有高孔隙率和低 导热性; (2) 聚吡咯(PPy)层(相当于叶子中的叶绿素层)负载于PVA海绵 表面以吸收太阳能; (3) 微/纳米尺度的多孔疏水表面层(相当于叶片中 的气孔层). 其中, 亲水海绵的PVA聚合物网络提供了高效水传输通道 的同时实现了内部水蒸发焓的降低; PPy层吸收太阳能并转化为内能加 热气液蒸汽界面的水, 实现热局部化, 大大减小了热损耗; 疏水性多孔 表面层的边缘效应, 为水蒸气提供了高效逃逸通道. 基于仿生叶片结构 的太阳能蒸汽转化装置在一倍太阳光强 $\left(1 \mathrm{~kW} \mathrm{~m}^{-2}\right)$ 下蒸发率高达 $3.09 \mathrm{~kg} \mathrm{~m}^{-2} \mathrm{~h}^{-1}$. 在室外自然光照条件下成功地对盐水和海水进行了脱 盐和净化, 平均净水速率为 $1.4 \mathrm{~L} \mathrm{~m}^{-2} \mathrm{~h}^{-1}$. 这种基于仿生叶片结构的太 阳能蒸汽转化装置提供了一种极具潜力的高效水净化方法, 有助于利 用可持续能源缓解全球水危机. 